SWEDISH SCHOOL OF ECONOMICS

AND BUSINESS ADMINISTRATION

THE YRJÖ JAHNSSON WORKING PAPER SERIES

IN INDUSTRIAL ECONOMICS

6 (2004)

Oz Shy \& Rune Stenbacka

SERVICE HOURS WITH ASYMMETRIC

DISTRIBUTIONS OF IDEAL SERVICE TIME 
Service Hours With Asymmetric Distributions of Ideal Service Time

Key words: Service Hours, Service Hour Competition, Nonuniform distribution of ideal service time

JEL Classification: L81, M2, J51

(C) Swedish School of Economics and Business Administration, Oz Shy \& Rune Stenbacka

Oz Shy \& Rune Stenbacka

Swedish School of Economics and Business Administration

P.O.Box 479

00101 Helsinki, Finland

Distributor:

Library

Swedish School of Economics and Business Administration

P.O.Box 479

00101 Helsinki

Finland

Phone: +358-9-43133 376, +358-9-43133265

Fax: $\quad+358-9-43133425$

E-mail: publ@hanken.fi

http://www.hanken.fi/hanken/eng/page1579.php

SHS intressebyrå IB (Oy Casa Security Ab), Helsingfors 2004

ISBN 951-555-858-1

ISSN 0424-7256 


\title{
Service Hours With Asymmetric Distributions of Ideal Service Time*
}

\author{
$\mathrm{Oz} \mathrm{Shy}^{\dagger}$ \\ University of Haifa, and \\ Swedish School of Economics, and \\ WZ-Berlin
}

\author{
Rune Stenbacka ${ }^{\ddagger}$ \\ Swedish School of Economics \\ and HECER
}

October 13, 2004

\begin{abstract}
We show that private provision of service hours will be inefficiently low from a social point of view independently of the market structure. We study how asymmetric distributions of ideal service time impact on this market failure. We establish that the time gap between any two opening and closing hours in an oligopoly equilibrium is constant and that this time gap increases if the distribution of ideal service times becomes more uniform. Finally, we establish that longest available service hours are sensitive to price changes but are invariant to changes in the market structure alone.
\end{abstract}

Keywords: Service Hours, Service Hour Competition, Nonuniform distribution of ideal service time

JEL Classification Numbers: L81, M2, J51

(Draft $=$ hoursmonop45.tex 2004/10/13 10:39)

*We are thankful for constructive comments from Paul Dobson, Staffan Ringbom and Michael Waterson. Financial support from The Yrjö Jahnsson Foundation and The Hanken Foundation is gratefully acknowledged. Part of this research was conducted while Rune Stenbacka was visiting WZB, whose hospitality is gratefully acknowledged.

†WZB - Social Science Research Center Berlin, Reichpietschufer 50, D-10785 Berlin, Germany. E-mail: ozshy@ozshy.com

$\ddagger$ Department of Economics, Swedish School of Economics, P.O. Box 479, 00101 Helsinki, Finland. E-mail: rune.stenbacka@hanken.fi 


\section{Introduction}

The performance of many industries, in particular all the service industries, is importantly affected by the determination of service hours. Short service hours typically save costs for service operators, but short business hours hurt consumers, who may face disutility associated with having to advance or postpone their business transactions relative to their ideal time. Business hours have always constituted a central issue that generates public debates no matter whether we focus on private industries or industries with heavy public involvement. For example, in the private retailing industry, business hours have traditionally been severely regulated in many European countries and more recently the effects of business hour liberalization have generated heated debates in the political arena as well as in the academic literature (see, for example, Clemenz $(1990,1994)$, Kay and Morris (1987), Inderst and Irmen (forthcoming), Tangay, Vallee and Lanoie (1995) and Thum and Weichenrieder (1997)). Similarly, no matter whether the transportation services in metropolitan areas are outsourced to private operators or not, the local policymakers have to determine the service hours and frequency of service.

In general, the determination of service hours is affected by at least four major considerations: (a) There are typically important spillovers between periods. This means that some consumers may advance (postpone) their service acquisition to the previous (next) period if the service is not available at their ideal time. (b) There is typically an asymmetric distribution of consumers' ideal service time, which leads to regular fluctuations between phases of high demand and low demand within each period. (c) The technology for service provision may exhibit increasing, constant or decreasing returns to scale in both sales and service hours. Finally, (d) price competition may be influenced by service hours. The novelty of our present approach is the focus on the first two aspects by assuming that the consumers' ideal service time follows an asymmetric distribution on the unit circle. In particular, we explore the relationship between market structure, service hour competition, and social welfare for a class of asymmetric distributions of ideal service time. Further, we will focus on service hour provision in isolation from its possible implications for price competition, because those issues have recently been analyzed by Inderst and Irmen (forthcoming) 
as well as Shy and Stenbacka (2004) within the context of duopolistic retail industries for a given unchanging distribution. In particular, in the present paper we explicitly explore the relationship among classes of ideal service time distributions, market structure, service hour competition, and social welfare.

Our analysis initially focuses on a private single operator. We characterize how the single operator's service hour provision depends on the degree of asymmetry of the distribution of consumers' ideal service time. We demonstrate that the private service provider will maintain inefficiently short service hours from a social point view. Subsequently, we introduce competition with respect to service hour provision.

For a duopolistic industry we characterize two feasible equilibrium configurations. First we find that the duopoly equilibrium, where the providers maintain symmetric service hours around the peak, has the feature that the service providers maintain different opening hours: One operator opens earlier and closes later than its rival. Such an asymmetric equilibrium emerges because there is a high return to a service operator from extending the hours relative to the rival, because such an operator can automatically capture all those consumers who postpone or advance their service acquisition. For such an equilibrium configuration we find that an increased degree of uniformity of the distribution of ideal service times induces an increased degree of differentiation with respect to the service hours. In this equilibrium configuration the service hour provision by the operator providing long service hours is shown to coincide with the service hours offered by a monopoly. We also characterize an alternative equilibrium configuration with service hours asymmetrically around the peak. These two equilibrium configurations are found to be equivalent from the social point of view.

Finally, we investigate an oligopoly industry of service operators. We establish that in the oligopoly equilibrium the time gap between any two opening and closing hours is constant. Further, we prove that this time gap increases if the distribution of ideal service times becomes more uniform. Then, we conclude that the maximal service hours offered by the operator with the longest opening hours is invariant to the market structure of service operators. This invariance result implies that the social benefit of competition is realized at the price dimension and possibly 
at the variety dimension, but not at the dimension represented by the longest available service hours.

We are not aware of any existing research contribution, which analyzes the socially optimal service hours, or the relationship between market structure and service hour provision, from the point of view of a public economics perspective. There are some related studies of asymmetric distributions of consumers' ideal variety within the field of industrial economics. Neven (1986) has explored some consequences for location equilibria of a non-uniform distribution of consumers within the framework of a Hotelling model of horizontal product differentiation. Also, Waterson (1990) has explored the consequences of asymmetries in the costs of supplying customers at different points of the tastes spectrum, for the profitability of an oligopolistic industry within the framework of horizontal product differentiation.

The present study is organized as follows. Section 2 develops a model of consumers who differ in their ideal service time. Section 3 analyzes the profit-maximizing service hours of a single service operator. Section 4 solves for the socially optimal service hours. Section 5 extends the model to two competiting service providers. Section 6 extends the model to any arbitrary number of service operators competing with respect to service hours. Section 7 explores some features associated with a price equilibrium. Finally, Section 8 offers concluding comments.

\section{The Model}

Consider a single service provider, for example a store, operating in a given area (the "firm" in what follows). What we have in mind here is a firm that provides a service, or sells a good, such that consumers attach utility to the precise time at which the service is provided or delivered. This means that the consumers' utility is affected by a store's opening hours, a bank's service hours and so on. Clearly, it seems that all services belong to this category. Our model also fits more regulated industries, such as local bus companies with a decreased service of operation during late night hours.

Time is indexed continuously on the unit circle. The unit circle representation is important 
as it formalizes the idea that there are spillovers between time periods. The most obvious interpretation of this circle is simply that of one day. Let $t_{o}$ and $t_{c}$ denote the opening and closing hours as determined by the service provider, where $0 \leq t_{o}<t_{c} \leq 1$. Figure 1 illustrates possible service hours maintained by this service provider.
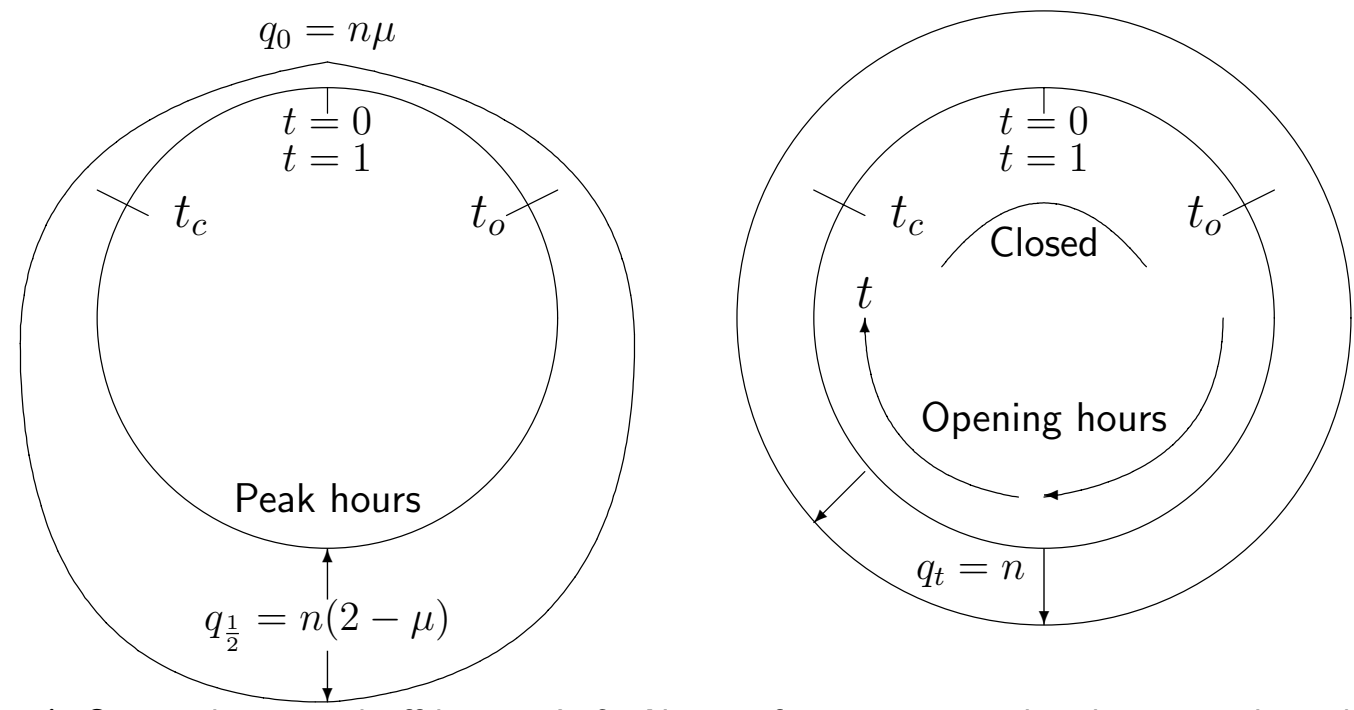

Figure 1: Service hours and off hours. Left: Non-uniform customer distribution with peak demand at $t=\frac{1}{2}(0<\mu<1)$. Right: Uniform distribution of consumers $(\mu=1)$.

\subsection{Consumer preferences}

Consumers are heterogeneous with respect to their most preferred time of consumption. For example, the optimal time of when to shop is distributed across the set of consumers. Each point in time $t$ on the unit circle $(0 \leq t \leq 1)$ represents an ideal service time for a consumer indexed by $t$. Let $p$ denote the price of the service/good provided by this firm. The utility of a consumer whose ideal service time is $t$ is given by

$$
U_{t} \stackrel{\text { def }}{=} \begin{cases}\beta-p & t \in\left[t_{o}, t_{c}\right] \\ \beta-p-\tau \min \left\{\left|t_{o}-t\right|,\left|t-t_{c}\right|\right\} & t \notin\left[t_{o}, t_{c}\right] \\ 0 & \text { Does not buy this service }\end{cases}
$$

where $|x-y|$ should be interpreted as the shortest arc distance between two points $x$ and $y$ on the unit circle. The parameter $\beta$ measures a consumer's basic utility derived from the consumption 
of the service. The parameter $\tau>0$, which we refer to as the value-of-time parameter, measures the per-unit-of-time disutility experienced by consumers when they are forced to either advance or postpone their service acquisition, because the service is not offered at their ideal time. We assume that the consumers are equally flexible when it comes to advancing or postponing the acquisition of this service. Observe that when the ideal for the service falls outside of the service hours, the consumer has two options: Either to postpone buying until service is resumed at $t_{o}$, or to advance buying to the closing time $t_{c}$.

In order to be able to distinguish between consumers who obtain service at their ideal time, and those who do not, let $\hat{t}_{o}$, where $\hat{t}_{o}<t_{o}$, denote a consumer who is indifferent between postponing purchasing the service to $t_{o}$, and not purchasing it at all. Similarly, let $\hat{t}_{c}$, where $\hat{t}_{c}>t_{c}$, denote a consumer who is indifferent between advancing purchasing the service to the closing hour $t_{c}$ and not buying at all. Figure 2 illustrates the types of consumers who obtain service at their ideal time, as well as consumers who must either postpone or advance their purchase.

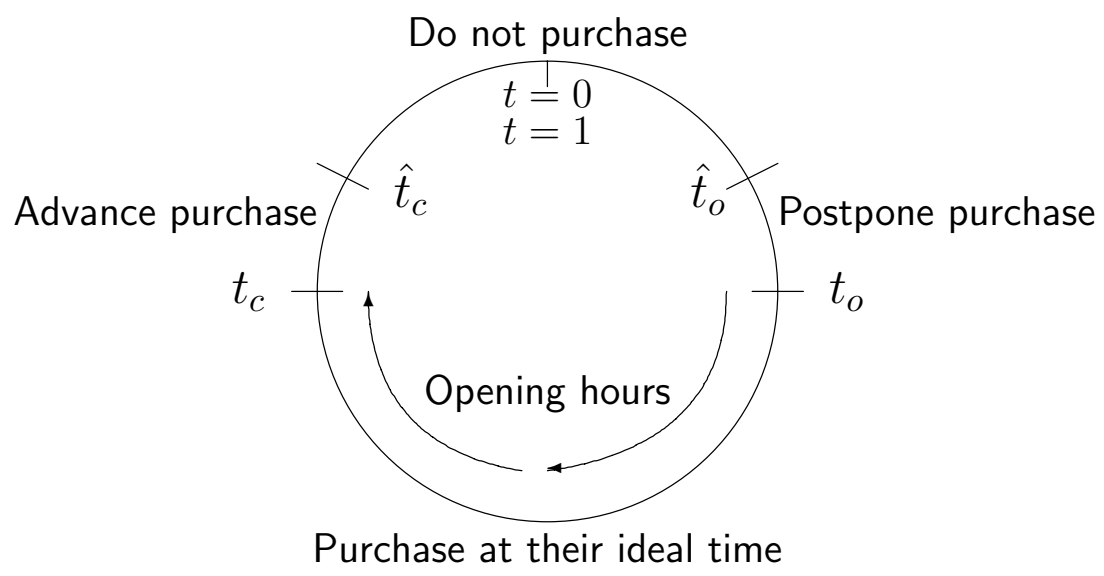

Figure 2: Consumers who obtain service at their ideal time, consumers with delayed or advanced service, and those who are not served at all.

\subsection{Consumer distribution}

In this paper, we wish to compute the service hours under a variety of asymmetric customer distributions, which include peak and off-peak phases. For this reason, we will analyze a general 
class of distribution functions in which the number of consumers with an ideal service time $t$ is

$$
q_{t} \stackrel{\text { def }}{=} \begin{cases}n[\mu+4(1-\mu) t] & \text { for ideal time } 0 \leq t \leq \frac{1}{2} \\ n[4-3 \mu-4(1-\mu) t] & \text { for ideal time } \frac{1}{2}<t<1\end{cases}
$$

where $0 \leq \mu \leq 1$, measures the degree of uniformity. More precisely, if $\mu=1$ then $q_{t}=n$ for all $t$, meaning that each time is an ideal service time for an equal number, $n$, of customers. That is, $\mu=1$ implies that the distribution of ideal service time is uniform. Figure 1 (left) illustrates a non-uniform distribution with a peak at $t=\frac{1}{2}$, and off-peak at $t=0$. As $\mu$ increases, the distribution becomes more uniform as illustrated in Figure 1(right). In contrast, as $\mu$ declines towards zero, the number of customers with an ideal service time at $t=0$ vanishes, whereas the number of peak customers at $t=\frac{1}{2}$ is maximized reaching the level of $q_{\frac{1}{2}}=2 n$.

In a technical sense, the main justification for focusing on the distribution function (2) is that it represents a "pure" shift in the degree of asymmetry, since any change in $\mu$ is independent of the population parameter $n$. More precisely, the total number of buyers (ideal service time) during the time intervals $\left[0, \frac{1}{2}\right]$ and $\left[\frac{1}{2}, 1\right]$ is constant and is given by

$$
\int_{0}^{\frac{1}{2}} q_{t} d t=n \int_{0}^{\frac{1}{2}}[\mu+4(1-\mu) t] d t=\int_{\frac{1}{2}}^{1} q_{t} d t=n \int_{\frac{1}{2}}^{1}[4-3 \mu-4(1-\mu) t] d t=\frac{n}{2} .
$$

Furthermore, through the introduction of the parameter $\mu$ in (2), we are formally able to characterize how asymmetries in the distribution of the consumers' ideal service time affect the endogenously-determined service hours.

We will make use of the following assumption.

\section{Assumption 1}

Consumers value their time. Formally,

$$
\tau>\frac{4(1-\mu)}{2-\mu}(\beta-p)
$$

Assumption 1 can be easily interpreted by looking at extreme distributions. If $\mu=1$ (uniform distribution), Assumption 1 becomes $\tau>0$. If $\mu=0$, this condition becomes $\tau / 2>(\beta-p)$, 
which must be assumed in order to avoid the trivial solution where the store is open only for a short time around $t=\frac{1}{2}$ while serving the entire consumer population. If Assumption 1 is reversed, consumers whose ideal time of service is half-a-day later or earlier would be willing to wait or advance their service time. For these consumers, the cost of waiting or advancing would be $\tau / 2$ whereas the benefit from obtaining the service would be $(\beta-p)$.

Next, we will be using the following terminology.

\section{DEFINITION 1}

We say that consumers are inflexible if they have a high value of time, formally if $\tau>4(\beta-p)$. We say that consumers are flexible if they have a low value of time, formally if

$$
\frac{4(1-\mu)}{2-\mu}(\beta-p)<\tau \leq 4(\beta-p) .
$$

Intuitively, Definition 1 implies that if consumers have a high value of time, they will never advance or postpone obtaining the service for more than $\frac{1}{4}$ of a day as their benefit from the service $\beta-p$ is smaller than the time cost $\tau / 4$.

\subsection{The service provider}

The service provider controls two strategic instruments: The opening hour, $t_{o}$, and the closing hour, $t_{c}$. On the cost side, we assume that the service technology exhibits constant returns to scale with a constant marginal cost $c$. In addition, we assume that the cost of operating the business is $w$ per unit of time. Therefore, the total cost of operation (independently of the sales level) is $w\left(t_{c}-t_{o}\right)$. We will refer to $w$ as the per-unit of time cost of business operation.

\section{Equilibrium Service Hours of a Single Service operator}

The environment discussed in the previous section extends Salop (1979) in the sense that it allows for a non-uniform distribution of consumer tastes. However, we found out that closed form solutions with respect to service hours and prices are impossible to obtain for a general class of asymmetric distributions of ideal service times. For this reason we confine our analysis 
to a fixed price $p .{ }^{1}$ Clearly, whenever we treat $p$ as fixed, we must assume that $\beta \geq p \geq c$, so that the price does not exceed consumers' willingness to pay, and is not below the marginal cost. Section 7 points out the difficulties of solving for price equilibria.

Figure 1(left) plots the consumer distribution defined in (2) and demonstrates that the offpeak hours are symmetrically distributed around $t=0$. Hence, a profit-maximizing service provider would choose its service hours to satisfy $t_{c}=1-t_{o}$. The utility function (1) implies that the consumer indifferent between postponing the service deal or not buying at all, indexed by $\hat{t}_{o}$, is determined by the equation $0=\beta-p-\tau\left(t_{o}-\hat{t}_{o}\right)$. Similarly, the location, $\hat{t}_{c}$, denoting the consumer indifferent between advancing and not buying at all, is determined by $0=\beta-p-$ $\tau\left(\hat{t}_{c}-t_{c}\right)$. Therefore,

$$
\hat{t}_{o}=t_{o}-\frac{\beta-p}{\tau} \text { and } \hat{t}_{c}=t_{c}+\frac{\beta-p}{\tau} .
$$

Clearly, a higher basic valuation parameter $\beta$ decreases $\hat{t}_{o}$ and increases $\hat{t}_{c}$, hence increases consumer participation. In contrast, an increase in the service price $p$ increases the range of excluded consumers.

Next, since the distribution of ideal service time (2) is symmetric around $t=\frac{1}{2}$, we will be solving for an equilibrium where

$$
t_{o}=1-t_{c} \quad \text { and } \quad \hat{t}_{o}=1-\hat{t}_{c} .
$$

This implies that the length of the service hours, when the service is offered, is $t_{c}-t_{o}=1-2 t_{o}$. Hence, Figure 2 and (5) imply that total output, which equals the total number of buyers, is

$$
Q\left(\hat{t}_{o}, \hat{t}_{c}\right)=n \int_{\hat{t}_{o}}^{\frac{1}{2}}[\mu+4(1-\mu) t] d t+n \int_{\frac{1}{2}}^{\hat{t}_{c}}[4-3 \mu-4(1-\mu) t] d t=2 n \int_{\hat{t}_{o}}^{\frac{1}{2}}[\mu+4(1-\mu) t] d t
$$

in a symmetric equilibrium where $t_{o}=1-t_{c}$.

\footnotetext{
${ }^{1}$ Stenbacka and Tombak (1995) made a similar assumption in their analysis of time-based competition, i.e. competition based on service speed, within the framework of a vertical differentiation model, which exhibits congestion effects.
} 
Substituting (4) into (6) yields the number of served consumers as a function of the opening hour only. Hence,

$$
Q\left(t_{o}\right)=n\left\{1-2\left(\frac{\beta-\tau t_{o}-p}{\tau}\right)\left[\mu-2(1-\mu)\left(\frac{\beta-\tau t_{o}-p}{\tau}\right)\right]\right\} .
$$

Thus, the number of excluded consumers is

$$
n-Q\left(t_{o}\right)=n\left\{2\left(\frac{\beta-\tau t_{o}-p}{\tau}\right)\left[\mu-2(1-\mu)\left(\frac{\beta-\tau t_{o}-p}{\tau}\right)\right]\right\} .
$$

Next, the cost of maintaining $t_{c}-t_{o}$ hours of operation is $w\left(t_{c}-t_{o}\right)=w\left(1-2 t_{o}\right)$, again by symmetry. Altogether, the service provider takes the price $p$ as given, and chooses an opening hour $t_{o}$ to solve

$$
\max _{t_{o}} \pi=(p-c) Q\left(t_{0}\right)-w\left(1-2 t_{o}\right),
$$

where $Q$ is given in (7). Maximizing (9) with the respect to the opening hour $t_{o}$ yields

$$
t_{o}=1-t_{c}=\frac{n(p-c)[4(1-\mu)(\beta-p)-\mu \tau]+w \tau}{4 n \tau(1-\mu)(p-c)} .
$$

We can show that

$$
t_{o} \leq \frac{1}{2} \quad \text { if } \quad w \leq\left. w\right|_{t_{o}=\frac{1}{2}} \stackrel{\text { def }}{=} \frac{1}{\tau} n(p-c)[(2-\mu) \tau-4(1-\mu)(\beta-p)] .
$$

That is, the operating cost parameter must be below a certain level, specified in (11), to ensure that the store is not always closed (the case where $t_{0}=\frac{1}{2}$ ). Observe that Assumption 1 implies that $\left.w\right|_{t_{o}=\frac{1}{2}}>0$. Also, note that the second-order condition for a maximum is $\mathrm{d}^{2} \pi / \mathrm{d} t_{o}^{2}=$ $8 n(1-\mu)(c-p)<0$ as the price was assumed to exceed marginal cost.

Next, by substituting the opening hour (10) into (4), we find that the buyers who are indifferent between postponing their service to the opening hour $t_{o}$ and not consuming at all, are indexed by

$$
\hat{t}_{o}=1-\hat{t}_{c}=\frac{w-n \mu(p-c)}{4 n(p-c)(1-\mu)} \geq 0 \quad \text { if } \quad w \geq\left. w\right|_{t_{o}=0} \stackrel{\text { def }}{=} n \mu(p-c) .
$$

Otherwise, if $w<n \mu(p-c), \hat{t}_{o}=1-\hat{t}_{c}=0$ meaning that all consumers are served. Notice that $w<n \mu(p-c)$ implies that it is profitable to operate even the most off-peak time period when consumer density is at its lowest level and is given by $\mu n$ (see Figure 2). This condition 
is interesting since $\hat{t}_{o}=1-\hat{t}_{c}=0$ does not mean that the service provider must operate at $t=0$. That is, the consumers located in the neighborhood of $t=0$ can advance or postpone their service time to the opening or closing hours.

Our comparative statics analysis will make sense only for interior equilibria where $0<\hat{t}_{o}<$ $t_{o}<\frac{1}{2}$ implying that some consumers are not served, and that the store is not always closed $\left(t<\frac{1}{2}\right)$. To obtain these interior equilibrium configurations, (11) and (12) imply that the operation cost parameter should be restricted as follows:

\section{Assumption 2}

The operating cost parameter is bounded from below and from above, so that the store is open but not full time. Formally,

$$
n \mu(p-c)=\left.w\right|_{t_{o}=0}<w<\left.w\right|_{t_{o}=\frac{1}{2}}=\frac{1}{\tau} n(p-c)[(2-\mu) \tau-4(1-\mu)(\beta-p)] .
$$

It is worth noting that when $\mu \rightarrow 1$, the range defined in Assumption 2 collapses to a singleton where $w=n(p-c)$. This means that under $\mu=1$ (uniform distribution), if $w>n(p-c)$ the store never opens, and if $w<n(p-c)$ the store is open nonstop. In this respect the asymmetric distribution of consumers is a crucial aspect of our model as the description of the optimal service hours converges to such a trivial configuration in the limiting case of the uniform distribution.

To compute the equilibrium profit, substitute (10) into (9) for $t_{o}$ to obtain

$$
\pi=\frac{(2-\mu)^{2} \tau n^{2}(p-c)^{2}+2 n w[4(\beta-p)(1-\mu)-\tau(2-\mu)](p-c)+w^{2} \tau}{4 n \tau(1-\mu)(p-c)} .
$$

We now turn to analyzing how opening hours are affected by the parameters of the model. Perhaps, the most interesting investigation is to alter the distribution of consumers' ideal service time, that is, changing the parameter $\mu$ in (2). We will make use of the following terminology.

\section{DEFINITION 2}

We say that the operating cost, as reflected by the parameter $w$, is high relative to the operating profit if $w>w^{H M} \stackrel{\text { def }}{=} n(p-c)$; at a medium level if $n(p-c) \sqrt{\mu(2-\mu)} \stackrel{\text { def }}{=} w^{M L} \leq w \leq w^{H M}$. Otherwise, if $w<w^{M L}$, we say that the operating cost is low. 
Observe it is never profitable to provide service around the clock if the operating cost is high, since the operating profit given by $n(p-c)$ does not cover the cost of nonstop operation given by $1 \times w$.

We now list the main findings of this section. Proofs are relegated to Appendix A.

\section{Proposition 1}

Suppose that the distribution of ideal service time (2) becomes more uniform ( $\mu$ increases). Then,

(a) (i) Opening hours expand if the operating cost is not high.

Formally, $\mathrm{d} t_{o} / \mathrm{d} \mu<0$ whenever $w<w^{H M}$.

(ii) Opening hours contract if the operating cost is high.

Formally, $\mathrm{d} t_{o} / \mathrm{d} \mu>0$ whenever $w>w^{H M}$.

(b) The number of served consumers increases if and only if the operating cost is low.

More precisely,

$$
\frac{\mathrm{d} Q}{\mathrm{~d} \mu} \geq 0 \quad \text { if and only if } \quad w \leq w^{M L}=n(p-c) \sqrt{\mu(2-\mu)} .
$$

(c) Profit always declines. Formally, $\mathrm{d} \pi / \mathrm{d} \mu<0$.

Part (c) is rather straightforward. To explain part (c) let us look at the opposite extreme situation where all consumers have their ideal service time at $t=\frac{1}{2}$ (i.e., a mass). Then, operation costs are zero, whereas all consumers are served, hence profit reaches the highest possible level. Part (a) shows that a more uniform distribution of ideal service time generates longer or shorter service hours depending on whether the cost of operation is high or not. Intuitively, this result formalizes the idea that the profit-maximizing service hours are extended when the distribution of customers becomes more uniform if the cost of operation is below the high level, $w^{\mathrm{HM}}$. However, for very high costs of operation the service hours are shortened, because in that case the firm has to concentrate its operation so as to exploit the peak in demand and the opportunities for doing this are reduced with a more uniform distribution of consumers. The result incorporated in part (b) can be interpreted along similar lines with the qualification that it covers the number of served customers instead of the service hours. 
Figure 3 illustrates our Proposition 1.

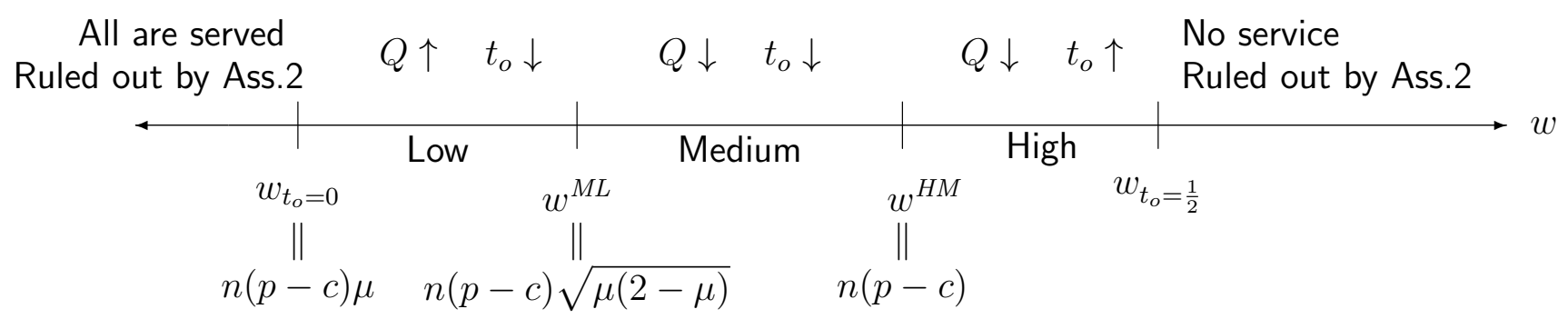

Figure 3: Illustration of Proposition 1: The effects of a more uniform distribution $(\mu \uparrow)$, as functions of the operation cost parameter $w$. Note: The range marked as High may be empty (Corollary 2).

The following corollary, proved in Appendix B, places a condition on buyers' value of time that is necessary to obtain the result listed in Proposition 1(a)(ii).

\section{Corollary 2}

A high value of time (see Definition 1) is a necessary condition for having a more uniform distribution contracting the service hours.

It is instructive to summarize this section with a numerical example confirming the results listed in Proposition 1. Table 1 displays some computations of the opening time $t_{0}$, the marginal customer type $\hat{t}_{o}$, the number of served consumers $Q$, and the equilibrium profit $\pi$.

The upper part Table 1 clearly demonstrates that as $\mu$ increases, $t_{o}$ increases (shorter service hours), and compared with the lower two parts, this can happen only when the value of time is high, as established in Corollary 2. Table 1 confirms that profit always declines when $\mu$ increases. In addition, the Table confirms that the number of served customers $Q$ declines for high values of $w$ and increases for low values of $w$. The two right columns demonstrate that the assumed values of $w$ indeed fall at the range of high, medium, and low operating costs as stated in Definition 2. The column third from the right confirms that $w$ falls in the range where the store maintains some opening hours as defined by $w_{t_{o}=\frac{1}{2}}$ in (11). 


\begin{tabular}{|c|c|c|c|c|c|c|c|}
\hline \multicolumn{8}{|c|}{ High $\tau=60 \&$ High operating cost: $w=1250>w^{H M}$} \\
\hline$\mu$ & $t_{0}$ & $\hat{t}_{0}$ & $Q$ & $\pi$ & $w_{t_{o}=\frac{1}{2}}$ & $w^{H M}$ & $w^{M L}$ \\
\hline 0.4 & 0.467 & 0.276 & 61.45 & 656 & $1344^{2}$ & 1200 & 960 \\
\hline 0.5 & 0.471 & 0.271 & 58.25 & 626 & 1320 & 1200 & 1039 \\
\hline \multicolumn{8}{|c|}{ Low $\tau=40 \&$ Medium operating cost: $\quad w=1050>w^{M L}$} \\
\hline$\mu$ & $t_{0}$ & $\hat{t}_{0}$ & $Q$ & $\pi$ & $w_{t_{o}=\frac{1}{2}}$ & $w^{H M}$ & $w^{H L}$ \\
\hline 0.4 & 0.498 & 0.198 & 74.76 & 892 & $1056^{2}$ & 1200 & 960 \\
\hline 0.5 & 0.487 & 0.187 & 74.22 & 864 & 1080 & 1200 & 1039 \\
\hline \multicolumn{8}{|c|}{ Low $\tau=40 \&$ Low operating cost: $\quad w=900<w^{M L}$} \\
\hline$\mu$ & $t_{0}$ & $\hat{t}_{0}$ & $Q$ & $\pi$ & $w_{t_{o}=\frac{1}{2}}$ & $w^{H M}$ & $w^{M L}$ \\
\hline 0.4 & 0.446 & 0.146 & 83.23 & 901 & 1056 & 1200 & 960 \\
\hline 0.5 & 0.425 & 0.125 & 84.37 & 877 & 1080 & 1200 & 1039 \\
\hline
\end{tabular}

Table 1: Computer simulations demonstrating how the opening time, number of served customers, and profit are affected when buyers' distribution becomes more uniform $(\mu \uparrow)$. Simulations assume $n=100, \beta=24, p=12$, and $c=0$.

\section{Socially Efficient Service Hours}

This section computes the socially optimal opening and closing hours for our model. We then compare these to the equilibrium service hours computed in the previous section.

We define the social welfare function of the economy as the sum of consumer surplus and industry profit. Since prices are merely transfers from consumers to service providers, the social welfare function is given by

$$
W=2(\beta-c) \int_{\hat{t}_{o}}^{\frac{1}{2}} q_{t} d t-2 \tau \int_{\hat{t}_{o}}^{t_{o}^{*}} q_{t}\left(t_{o}^{*}-t\right) d t-w\left(1-2 t_{o}^{*}\right),
$$

where $q_{t}$ is given in the upper part of (2). The first term measures buyers' utility net of unit production cost. The second term measures the total adjustment costs of the consumers who have to advance or postpone their service acquisition so as the make transactions take place when service is available. The last term is the cost of maintaining $t_{c}^{*}-t_{o}^{*}=1-2 t_{o}^{*}$ service hours.

Substituting (4) into (14) for $\hat{t}_{o}$, and then differentiating with respect to $t_{o}^{*}$ shows that the 
socially optimal opening hour is

$$
t_{o}^{*}=\frac{w \tau-c n[4 p(\mu-1)+4 \beta(1-\mu)-\mu \tau]-n \beta[2 \beta(\mu-1)+\mu \tau]-2 p^{2}(\mu-1)}{4 n \tau(1-\mu)(\beta-c)} .
$$

Subtracting the socially optimal opening hour from the equilibrium hour given in (10) yields

$$
t_{o}-t_{o}^{*}=\frac{(\beta-p)[2 n(p-c)(1-\mu)(\beta-p)+w \tau]}{4 n \tau(1-\mu)(p-c)(\beta-c)}>0 .
$$

Hence,

\section{Proposition 3}

(a) A single service provider will maintain inefficiently short service hours from a social point of view. Formally, $t_{o}>t_{o}^{*}$ and $t_{c}<t_{c}^{*}$.

(b) The provider's profit-maximizing service hours equal the socially optimal level if the price is set at the level where all consumer surplus is extracted. Formally, $t_{o}=t_{o}^{*}$ if $p=\beta$.

Part (a) can be intuitively explained by reference to the fact that the private service provider does not internalize the social costs borne by those customers, who have to advance or postpone their business transaction. In this respect, and at least in the absence of price effects, all regulation which is designed to limit the service hours, seems to be misplaced as any potential economically justified regulation should impose requirements on minimum, not maximum, service hours. Proposition 3(b) is very much related to Swan's (1970a,b), where a price setting monopoly will use the price system to extract more surplus, but will maintain social optimum with respect to other decision variables (opening hours in the present case). Thus, a monopoly service provider has an incentive to utilize only the price, and not service hours, to extract surplus from consumers.

We now ask what is the relationship between the magnitude of the market failure characterized in Proposition 3 and the distribution of consumers' ideal service time? By differentiation of (16) with respect to $\mu$, and separately with respect to the price $p$, we find that

$$
\begin{aligned}
\frac{\mathrm{d}\left(t_{o}-t_{o}^{*}\right)}{\mathrm{d} \mu} & =\frac{w(\beta-p)}{4 n(p-c)(\beta-c)(1-\mu)^{2}}>0 \quad \text { and } \\
\left.\frac{\mathrm{d}\left(t_{o}-t_{o}^{*}\right)}{\mathrm{d} p}\right|_{c=0} & =\frac{-4 n p^{2}(\beta-p)(1-\mu)-w \beta \tau}{4 n p^{2} \beta \tau(1-\mu)}<0 .
\end{aligned}
$$


Therefore, we can state the following proposition.

\section{Proposition 4}

The difference between the socially-optimal and the equilibrium opening hours

(a) increases when the distribution becomes more uniform, and

(b) decreases with an increase in the price of the service.

Thus, our model predicts that the market failure associated with reduced service hours is less significant with a more asymmetric distribution of buyers' ideal service time. The distortion is magnified with a more uniform distribution of consumers. Intuitively this makes sense, because with a more uniform distribution a larger number of consumers have to adjust the service time of their business transaction.

Proposition 4(b) demonstrates that the shortage of service hours from a social viewpoint is smaller for high-priced services (with insignificant marginal costs). This implies the following corollary.

\section{Corollary 5}

In markets with low marginal service production cost, the introduction of price competition enlarges the difference between the business hour equilibrium and the socially optimal service hours.

The next section investigate whether competition in service hours (holding the price level constant) can serve as a mechanism for extending the service hours towards the social optimum.

\section{Equilibrium Service Hours under Duopoly}

Suppose now that there are two service providers labeled as 1 and 2. Let $t_{o}^{1}$ and $t_{c}^{1}$ denote the opening and closing time of service provider $1 . t_{o}^{2}$ and $t_{c}^{2}$ are similarly defined. We will distinguish between two equilibrium configurations. 


\section{DEFINITION 3}

We say that a service operator $i$ extends its opening hours symmetrically around the peak if the time interval between the opening hour and the peak hour equals the time interval between the peak hour and the closing hour. Formally, if $\frac{1}{2}-t_{o}^{i}=t_{c}^{i}-\frac{1}{2}, i=1,2$.

Section 5.1 analyzes symmetric service operations around the peak hour. Section 5.2 solves for equilibria with service hours asymmetrically located around the demand peak.

\subsection{Duopoly equilibrium with symmetric-around-the-peak service hours}

Figure 4 displays opening and closing hours which are symmetric around the demand peak assuming, with no loss of generality, that $t_{o}^{1} \leq t_{o}^{2}$ and $t_{c}^{1} \geq t_{c}^{2}$. Figure 4 shows that both firms

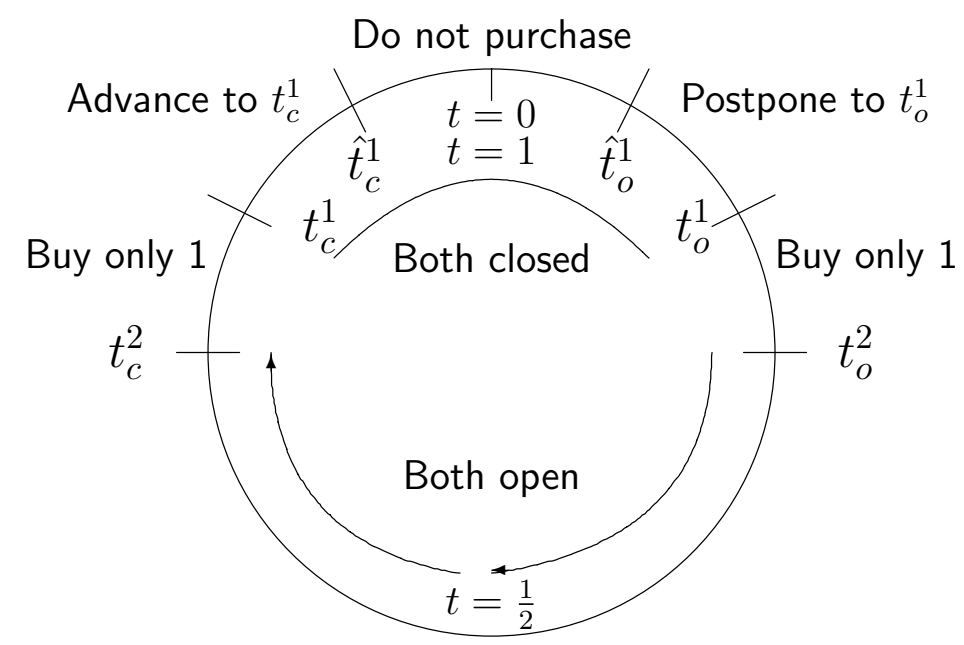

Equally split between $1 \& 2$

Figure 4: Two service providers: Symmetric around the peak opening hours.

provide service between $t_{o}^{2}$ and $t_{c}^{2}$. Firm 1 starts providing the service earlier, at $t_{o}^{1}$ and closes at a later time $t_{c}^{1}$. Therefore, given equal prices, all buyers indexed on $\left[\hat{t}_{o}^{1}, t_{o}^{1}\right)$ obtain the service later than their ideal time, and from firm 1 only, whereas all buyers indexed on $\left(t_{c}^{1}, \hat{t}_{c}^{1}\right]$ obtain their service also from firm 1 and earlier than their ideal time. 
Similar to (4), the buyers who are indifferent between obtaining the service at a non-ideal time and not obtaining it at all are indexed by

$$
\hat{t}_{o}^{1}=t_{o}^{1}-\frac{\beta-p}{\tau} \quad \text { and } \quad \hat{t}_{c}^{1}=t_{c}^{1}+\frac{\beta-p}{\tau} .
$$

In view of Figure 4, assuming that buyers split evenly between the providers when both stores are open, the number of clients served by each firm is

$$
q_{1}=2 n \int_{\hat{t}_{o}^{1}}^{t_{o}^{2}}[\mu+4(1-\mu) t] d t+2 \frac{1}{2} n \int_{t_{o}^{2}}^{\frac{1}{2}}[\mu+4(1-\mu) t] d t \quad \text { and } \quad q_{2}=2 \frac{1}{2} n \int_{t_{o}^{2}}^{\frac{1}{2}}[\mu+4(1-\mu) t] d t .
$$

The service providers choose the opening hours to solve

$$
\max _{t_{o}^{1}} \pi_{1}=(p-c) q_{1}-w\left(1-2 t_{o}^{1}\right) \quad \text { and } \quad \max _{t_{o}^{2}} \pi_{2}=(p-c) q_{2}-w\left(1-2 t_{o}^{2}\right),
$$

where $q_{1}$ and $q_{2}$ are given in (19) and $\hat{t}_{o}^{1}$ in (18). It can be shown that $\mathrm{d}^{2} \pi_{1} / \mathrm{d}\left(t_{o}^{1}\right)^{2}=8 n(1-$ $\mu)(c-p)<0$, and that $\mathrm{d}^{2} \pi_{2} / \mathrm{d}\left(t_{o}^{2}\right)^{2}=4 n(1-\mu)(c-p)<0$, hence both providers' profit functions are strictly concave with respect to their opening hours. Thus, the first-order conditions imply the following opening hours:

$$
t_{o}^{1}=\frac{n(p-c)[4(\beta-p)(1-\mu)-\mu \tau]+w \tau}{4 n \tau(1-\mu)(p-c)} \quad \text { and } \quad t_{o}^{2}=\frac{2 w-\mu n(p-c)}{4 n(1-\mu)(p-c)}
$$

Comparing (21) with (10) demonstrates that the opening hour of firm 1 in duopoly competition equals the opening hour of a single service provider. Thus, under duopoly, the service provider who opens earlier determines the opening hour by equating the per-unit-of-time operating cost $w$ with the marginal operating profit at the opening hour. This consideration is identical to the one made by a single provider, which is a consequence of our assumption that prices are fixed.

Why do two initially identical service providers end up maintaining different opening hours? The answer is that the store that opens longer hours attains two additional market segments: The early morning customers who postpone service to the opening hour, and late customers who advance their service acquisition. In contrast, the store with shorter service hours captures only customers at their ideal service times. Consequently, there is an additional return from 
maintaining longer hours and this additional return explains why identical opening hours does not constitute a Nash equilibrium. If the service provider with the shorter hours would match the opening hours of the long hour operator, this additional return would be cut by half and would not cover the additional operating costs.

The opening hours (21) imply that the difference between the two opening hours (hence, the duration at which only one firm provides the service) is

$$
t_{o}^{2}-t_{o}^{1}=\frac{w \tau-4 n(1-\mu)(\beta-p)(p-c)}{4 n \tau(1-\mu)(p-c)}>0 \quad \text { if } \quad w>\frac{4 n(1-\mu)(p-c)(\beta-p)}{\tau} .
$$

Appendix $\mathrm{C}$ provides the proof of the following proposition.

\section{Proposition 6}

Suppose that (22) holds and that the distribution of ideal service time becomes more uniform ( $\mu$ increases). Then,

(a) Both providers extend their service hours if $w<n(p-c) / 2=w^{H M} / 2$.

(b) Provider 1 extends whereas provider 2 shortens business hours if $n(p-c) / 2<w<n(p-c)$.

(c) Both providers shorten their service hours if $w>w^{H M}=n(p-c)$, that is if operating costs are high according to Definition 2.

(d) The gap between the opening and closing hours of the two providers is enlarged.

The reader can notice the resemblance between Proposition 6 and Proposition 1 which is also illustrated in Figure 3, as both firm 1 under duopoly competition, and a single provider offer identical service hours, and hence react to changes in the distribution in the same way via the parameter $w$. In fact, the condition listed in Proposition 6(a) and (b) can be logically deduced since each provider serves $50 \%$ of the customers, hence the threshold value equals precisely to $w^{H M} / 2$. For this reason we can omit a formal proof of this proposition. Part (d) means that an increased degree of uniformity of the distribution of ideal service time implies an increased degree of differentiation with respect to opening hours. This is a natural features because with a more uniform distribution a larger number of consumers will adjust their business transactions to take 
place during firm 1's opening hours. This, in turn, will increase firm 1's return from extended service hours. At the same time, firm 2 will face a lower number of peak-hour customers, which leads firm 2 to shorten its service hours.

Table 2 demonstrates our finding regarding the effect of a changing distribution on equilibrium service hours.

\begin{tabular}{|c|cccccc|}
\hline \multicolumn{8}{c|}{ Proposition 6(a): $w=550<600=w^{H M} / 2$} \\
\cline { 2 - 7 } & $\hat{t}_{0}^{1}$ & $t_{0}^{1}$ & $t_{o}^{2}$ & $t_{o}^{2}-t_{o}^{1}$ & $\pi_{1}$ & $\pi_{2}$ \\
0.2 & 0.080 & 0.173 & 0.224 & 0.051 & 326.62 & 146.30 \\
0.3 & 0.056 & 0.149 & 0.220 & 0.071 & 323.06 & 131.49 \\
0.4 & 0.024 & 0.117 & 0.215 & 0.099 & 323.31 & 116.74 \\
\hline \multicolumn{6}{c}{ Proposition 6(b): $w=650>600=w^{H M} / 2$} \\
$\mu$ & $\hat{t}_{0}^{1}$ & $t_{0}^{1}$ & $t_{o}^{2}$ & $t_{o}^{2}-t_{o}^{1}$ & $\pi_{1}$ & $\pi_{2}$ \\
0.2 & 0.107 & 0.199 & 0.276 & 0.077 & 326.33 & 96.30 \\
0.3 & 0.086 & 0.179 & 0.280 & 0.101 & 327.23 & 81.49 \\
0.4 & 0.059 & 0.151 & 0.285 & 0.133 & 333.44 & 66.74 \\
\hline
\end{tabular}

Table 2: Computer simulations showing how the opening hours, difference in opening hours, number of served customers, and profits are affected when buyers' distribution becomes more uniform $(\mu \uparrow)$. Simulations assume $n=100, \tau=130, \beta=24, p=12$, and $c=0$.

Table 2 verifies and demonstrates the results listed in Proposition 6. The top table demonstrates that the opening hours of firm 1 expand when operating expenses are sufficiently low. The bottom table demonstrates that for intermediate operating costs provider 2 shortens its service hours, whereas the large operator extends its service hours. Finally, Table 2 confirms Proposition $6(\mathrm{~d})$ showing that the gap between opening hours is enlarged with a more uniform distribution.

We now wish to investigate how service hours are affected by an exogenous increase in the price of the service. Differentiating (21) with respect to $p$ yields

$$
\frac{\mathrm{d} t_{o}^{1}}{\mathrm{~d} p}=-\frac{w}{4 n(1-\mu)(p-c)^{2}}-\frac{1}{\tau}<0 \quad \text { and } \quad \frac{\mathrm{d} t_{o}^{2}}{\mathrm{~d} p}=-\frac{w}{2 n(1-\mu)(p-c)^{2}}<0 .
$$

Hence, 


\section{Proposition 7}

Suppose that there is an increase in the price of the service, then

(a) Both service providers extend their service hours.

(b) The difference between the service hours of the two providers increases as long as the operating cost does not exceed a certain threshold level. Formally,

$$
\frac{\mathrm{d}\left(t_{o}^{2}-t_{o}^{1}\right)}{\mathrm{d} p} \geq 0 \quad \text { if and only if } \quad w \leq \frac{4 n(1-\mu)(p-c)^{2}}{\tau} .
$$

The intuition behind the first part is straight forward as it is clear that a higher price makes it more profitable to extend service hours. Part (b) states that firm 1 extends its hours "faster" than firm 2 for sufficiently low operating costs, and vice versa.

\subsection{Duopoly equilibrium with asymmetric-around-the-peak service hours}

Figure 5 displays asymmetric opening and closing hours assuming, with no loss of generality, that firm 1 opens earlier than firm 2 and also closes earlier than firm 2, that is $t_{o}^{1} \leq t_{o}^{2}$ and $t_{c}^{1} \leq t_{c}^{2}$. Comparing Figure 5 to Figure 4 reveals that both providers maintain the same opening hours. The difference between the figures is that in Figure 5 provider 1's closing time is earlier than that of provider 2. However, in Figure 5 both providers maintain the same length of opening hours (in contrast to Figure 4 where provider 1 maintains longer hours). Loosely speaking, Figure 5 "reverses' the roles of provider 1 and provider 2 during the second half of the day.

Clearly, since in this asymmetric equilibrium both service operators provide the same lengths of opening hours, they bear the same operation costs. Hence, there is no need to recalculate the opening and closing hours. Instead, we need only switch between $t_{c}^{1}$ and $t_{c}^{2}$, therefore,

$$
\begin{aligned}
& t_{c}^{1}=1-t_{o}^{2}=\frac{n(p-c)(4-3 \mu)-2 w}{4 n(1-\mu)(p-c)} \text { and } \\
& t_{c}^{2}=1-t_{o}^{1}=\frac{n(p-c)[\tau(4-3 \mu)-4(\beta-p)(1-\mu)]-w \tau}{4 n \tau(1-\mu)(p-c)},
\end{aligned}
$$

where $t_{o}^{1}$ and $t_{o}^{2}$ are given in (21). Now we can state the following proposition. 
Do not purchase

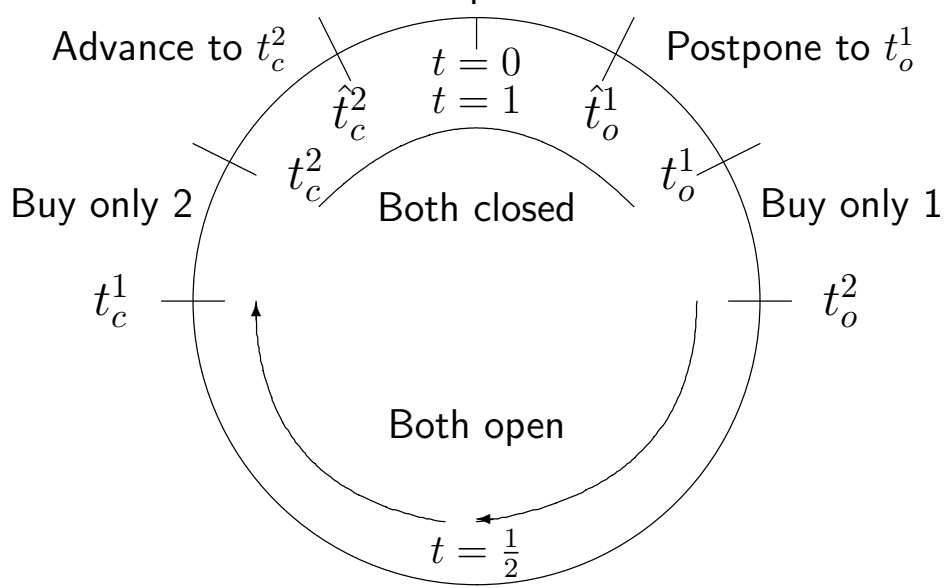

Equally split between $1 \& 2$

Figure 5: Two service providers: Asymmetric around the peak opening hours.

\section{Proposition 8}

(a) There exist two equilibria where the service operators extend their opening hours asymmetrically around the peak. (b) In both equilibria, one service provider opens early and closes early, whereas the competing provider opens late and closes late. (c) Both providers earn the same profits.

The asymmetric configuration captured by Proposition 8 could be exemplified by the opening hour pattern often applied by bars and night clubs. Night clubs tend to open late and close late.

It is important to emphasize that the symmetric and the asymmetric equilibrium configurations generate allocations, which are equivalent from a social point of view as long as the prices are fixed. Of course, in a more general context with endogenous prices it may matter a great deal from a social point of view whether the equilibrium configuration is symmetric or asymmetric around the peak. In such a generalized context, the asymmetric business hour configuration may facilitate market sharing and thereby serve as a collusion device. 


\section{Equilibrium Service Hours under Oligopoly}

Sections 3 and 5 analyzed the market structures with a single service provider as well as a duopoly. In this section we demonstrate how our model can be extended to incorporate any number of service providers. Perhaps the most interesting issue to investigate is the time gaps between the providers' opening (and closing) hours.

Suppose now that there are $\phi \geq 3$ service providers who all choose to be open around the peak time $t=\frac{1}{2}$. As before, we assume that the buyers are equally divided among all operators which are open. Figure 6 illustrates possible opening hours on the half-circle $\left[0, \frac{1}{2}\right]$.

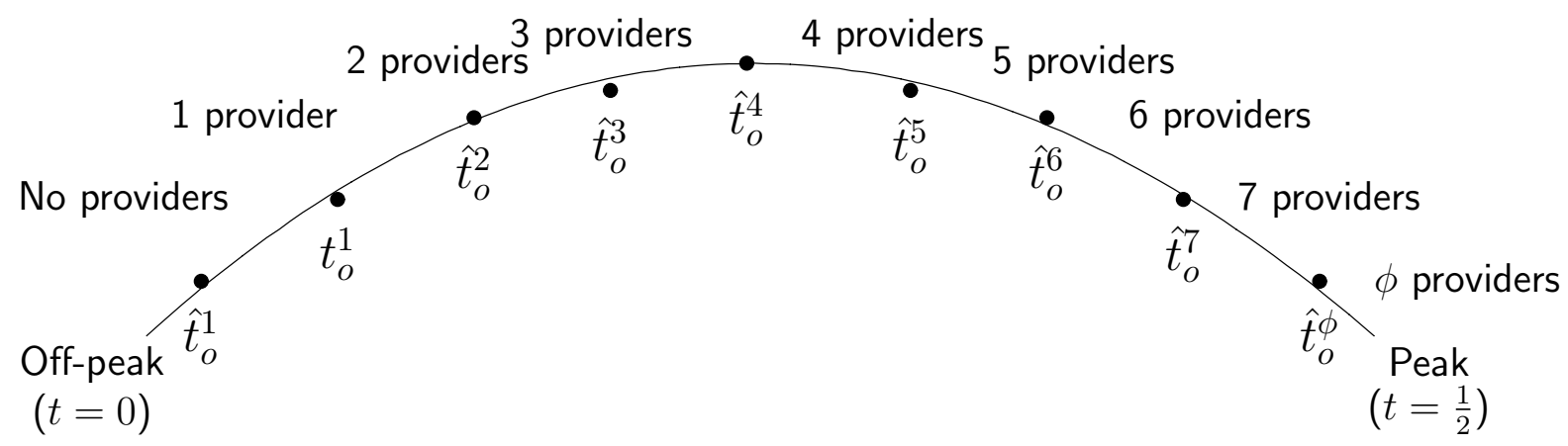

Figure 6: Equidistant opening hours under oligopoly.

Let $f(\mu, t) \stackrel{\text { def }}{=} \mu+4(1-\mu) t$. A straigtforward generalization of $(19)$ shows that the number of buyers served by firm $i$ (for $2 \leq i \leq \phi-1$ ) is

$$
q_{i}=\frac{2 n}{i} \int_{t_{o}^{i}}^{t_{o}^{i+1}} f(\mu, t) d t+\frac{2 n}{i+1} \int_{t_{o}^{i+1}}^{t_{o}^{i+2}} f(\mu, t) d t+\cdots+\frac{2 n}{\phi} \int_{t_{o}^{\phi}}^{\frac{1}{2}} f(\mu, t) d t .
$$

That is, consumers with an ideal time close to $t=\frac{1}{2}$ are divided among a larger number of providers, whereas buyers whose ideal service time is further away from $t=\frac{1}{2}$ are successively divided among fewer providers. Similar to (20), each of the above service providers chooses the opening hour $t_{o}^{i}$ to maximize $\pi_{i}=(p-c) q_{i}-w\left(1-2 t_{o}^{i}\right)$, where $q_{i}$ is given in (25). Abstracting from corner solutions, the opening hour of provider $i$ is given by

$$
t_{o}^{i}=\frac{i w-\mu n(p-c)}{4 n(1-\mu)(p-c)} \text {. }
$$


Since $\mathrm{d}^{2} \pi_{i} / \mathrm{d}\left(t_{o}^{i}\right)^{2}=8 n(1-\mu)(c-p)<0,(26)$ is a unique maximum. Differentiating (26) with respect to the distribution parameter $\mu$ and the price $p$ yields

$$
\frac{\mathrm{d} t_{o}^{i}}{\mathrm{~d} \mu}=\frac{i w-n(p-c)}{4 n(p-c)(1-\mu)^{2}} \quad \text { and } \quad \frac{\mathrm{d} t_{o}^{i}}{\mathrm{~d} p}=\frac{i w}{4 n(\mu-1)(p-c)^{2}},
$$

where the second expression is always negative. Therefore,

\section{Proposition 9}

(a) When the distribution becomes more uniform, service providers with longer service hours are more likely to extend their opening hours than providers with shorter service hours. Formally,

$$
\frac{\mathrm{d} t_{o}^{i}}{\mathrm{~d} \mu} \leq 0 \quad \text { if and only if } \quad i \leq \frac{n(p-c)}{w}
$$

(b) All service providers increase their service hours when the price increases.

Note that the firms indexed by a lower $i$ have longer service hours. Part (a) implies that a low value of the product $i \times w$ induces these firms to extend hours when the distribution becomes more uniform. This holds true, because making the distribution more uniform shifts buyers from firms indexed by a high $i$ with concentrated service hours towards firms with longer service hours. For this reason, part (a) captures the idea that firms indexed by high $i$ tend to reduce their service hours since they tend to lose buyers.

In order to gain further insights how opening and closing hours vary across oligopoly competitors, let us look at a provider indexed by $j<i$. Then, (26) implies that

$$
t_{o}^{i}-t_{o}^{j}=\frac{w(i-j)}{4 n(1-\mu)(p-c)} .
$$

In particular, setting $j=i+1$ yields the following proposition.

\section{Proposition 10}

(a) The time gap between any two opening and closing hours is constant. Formally, $t_{o}^{i+1}-t_{o}^{i}$ is independent of $i$, for any service provider $2 \leq i \leq \phi$.

(b) The time gap between any two opening hours (similarly, between any two closing hours) increases when the distribution becomes more uniform. 
Part (a) confirms the configuration illustrated in Figure 6, which exhibits opening hours at constant intervals. Of course, this result may be attributed to the assumption of the constant value of time parameter, $\tau$. The only exception is service provider 1 with the longest service hours. The reason is that firm 1 also serves buyers whose ideal service time falls when no firm provide any service. That is, firm 1 also serves buyers indexed on $\left[\hat{t}_{o}^{1}, t_{o}^{1}\right)$ and $\left[t_{c}^{1}, \hat{t}_{c}^{1}\right]$ during which no firm provides any service. Proposition 10 (b) captures the idea that a service operator with longer business hours has higher returns from extending its service hours further in response to a shift towards more off-peak customers. In this sense, a shift towards a more uniform distribution of consumers leads to a higher degree of business hour differentiation. Or, conversely, a change towards a more asymmetric distribution of consumers induces a tendency towards increased agglomeration of the service hours around the peak hour.

\section{Service Hours and Pricing Decisions}

Our analysis has so far assumed that the price of the service/good has been fixed. This could be the result of a regulatory requirement, or some kind of resale price maintenance marketing arrangements. In this section we "reverse" our assumption regarding the strategic decisions by holding the opening and closing hours constant and characterizing the price equilibrium. In particular, we are interested in finding out how the price equilibrium is affected by varying the (now) exogenously-given service hours.

Substituting (7) into (9), and then maximizing (9) with respect to $p$, taking $t_{o}$ as given, yields

$$
\begin{aligned}
p\left(t_{o}, \mu\right) & =\frac{\sqrt{A+B}+C}{6(1-\mu)}, \quad \text { where } \\
A & \stackrel{\text { def }}{=} 4 c^{2}(\mu-1)^{2}+2 c(\mu-1)\left(4 t_{o} \tau(\mu-1)+4 \beta(1-\mu)-\mu \tau\right)+4 t_{o}^{2} \tau^{2}(\mu-1)^{2} \\
B & \stackrel{\text { def }}{=} 2 t_{o} \tau(1-\mu)(4 \beta(\mu-1)+\mu \tau)+4 \beta^{2}(\mu-1)^{2}+2 \beta \mu \tau(\mu-1)+\tau^{2}(\mu-3 \mu+3) \\
C & \stackrel{\text { def }}{=} 2 c(1-\mu)+4 t_{o} \tau(\mu-1)+4 \beta(1-\mu)-\mu \tau .
\end{aligned}
$$

From this algebraic expression, the reader can already observe that although closed-form solutions for the price equilibrium and the associated profits can be obtained, systematic and general 
comparative statics are hard to display. Therefore, we here extract only some limited observations based on numerical simulations.

Table 3 demonstrates how prices, profits, and the number of buyers who postpone/advance the service transaction, are affected when service hours are shortened. ${ }^{2}$ From Table 3 we can

\begin{tabular}{|r|cccc|}
\hline$t_{o}$ & $p$ & $\pi$ & $\hat{t}_{o}$ & $t_{o}-\hat{t}_{o}$ \\
0.2 & 23.11 & 1190 & 0.182 & 0.0177 \\
0.3 & 19.69 & 1016 & 0.214 & 0.0862 \\
0.4 & 16.41 & 872 & 0.248 & 0.1518 \\
\hline
\end{tabular}

Table 3: Computer simulations showing how shortening service hours (increasing $t_{o}$ ) affects equilibrium price, profit, and threshold buyers. Simulations assume $n=100, \tau=50, \beta=24, c=0$, $\mu=0.4$, and $w=1000$.

extract the following list of results.

\section{Observation 11}

Suppose that service hours are shortened. Then, (a) the service provider reduces the price; (b)profit declines, and (c) the number of served customers declines, but at a decreasing rate.

Clearly, the service provider reduces the price in order to attract more people to advance/postpone their service time. That is, price is reduced to partially compensate for the loss of customers due to shortened service hours. This can be seen on the right column of Table 3 which shows that the number of consumers who advance/postpone service $t_{o}-\hat{t}_{o}$ increases because the price is lowered. The price reduction associated with shortened service hours also confirms the prediction by, for example, Inderst and Irmen (2004) that business hour deregulation tends to raise retail prices.

Finally, we now investigate how changing the distribution of consumers' ideal service time affects equilibrium prices and profit levels. Table 4 displays how these variables vary with the distribution parameter $\mu$.

Table 4 generates the following observations.

\footnotetext{
${ }^{2}$ Our simulations also verified that the second-order condition for a maximum is satisfied $\tau^{2} \mathrm{~d}^{2} \pi / \mathrm{d} p^{2}=$ $-4 n\left[2 c(\mu-1)+6 p(1-\mu)+4 t_{o} \tau(1-\mu)+4 \beta(\mu-1)+\mu \tau\right]<0$ at the optimized value of $p$.
} 


\begin{tabular}{|c|cccc|}
\hline$\mu$ & $p$ & $\pi$ & $\hat{t}_{o}$ & $t_{o}-\hat{t}_{o}$ \\
0.4 & 19.69 & 1016 & 0.214 & 0.0861 \\
0.5 & 19.35 & 969 & 0.207 & 0.0930 \\
0.6 & 18.97 & 922 & 0.199 & 0.1006 \\
\hline
\end{tabular}

Table 4: Computer simulations showing how making the distribution more uniform (increasing $\mu$ ) affects equilibrium price, profit, and threshold buyers. Simulations assume $n=100, \tau=50, \beta=24$, $c=0, t_{o}=0.3$, and $w=1000$.

\section{Observation 12}

Suppose that buyers' distribution of ideal service time becomes more uniform ( $\mu$ increases). Then, (a) equilibrium price and profit decrease; and (b) more consumers are served

The increase in the number of served consumers follows from two effects operating in the same direction: The decline in $p$ and the shift of consumers from peak to off-peak hours.

\section{Conclusion}

In this study we have theoretically established that private provision of service hours will be inefficiently low from a social point of view independently of the market structure. We have investigated how asymmetric distributions of ideal service time impact on this market failure. We have shown that the time gap between any two opening and closing hours in an oligopoly equilibrium is constant and that this time gap increases if the distribution of ideal service times becomes more uniform. Finally, we have established that maximal service hour provision is invariant to the market structure in the industry of service operators. This invariance result means that the social benefit of competition is realized at the price dimension and possibly at the variety dimension, but not at the dimension represented by the longest available service hours.

\section{Appendix A. Proof of Proposition 1}

Differentiating (10) and (12) with respect to $\mu$ yields

$$
\frac{\mathrm{d} t_{o}}{\mathrm{~d} \mu}=\frac{\mathrm{d} \hat{t}_{o}}{\mathrm{~d} \mu}=\frac{w-n(p-c)}{4 n}<0 \quad \text { if } \quad w<n(p-c)=w^{H M} .
$$


This proves part (a). To prove part (b), observe that

$$
\frac{\mathrm{d} Q}{\mathrm{~d} \mu}=\frac{\mu(2-\mu) n^{2}(p-c)^{2}-w^{2}}{4 n(1-\mu)^{2}(p-c)^{2}} \geq 0 \quad \text { if and only if } \quad w \leq w^{M L} .
$$

Finally, to prove (c),

$$
\frac{\mathrm{d} \pi}{\mathrm{d} \mu}=\frac{c^{2} n^{2} \mu(\mu-2)-2 c n[n p \mu(\mu-2)+w]+n^{2} p^{2} \mu(\mu-2)+2 n p w-w^{2}}{4 n(p-c)(1-\mu)^{2}}<0
$$

if $w>\mu n(p-c)$ which holds by Assumption 2 .

\section{Appendix B. Proof of Corollary 2}

As shown in Figure 3, this case happens when $w$ fall in the interval where $w^{H M}<w<\left.w\right|_{t=\frac{1}{2}}$. (11) and Definition 2 imply that this interval is nonempty if $\tau>4(\beta-p)$, which means high value of time according to Definition 1 .

\section{Appendix C. Proof of Proposition 6}

Part (c) is identical to Proposition 1(a). To prove (a), Differentiating (21) with respect to $\mu$ yields

$$
\frac{\mathrm{d} t_{o}^{2}}{\mathrm{~d} \mu}=\frac{2 w-n(p-c)}{4 n(1-\mu)^{2}(p-c)} \leq 0 \quad \text { if } \quad w \leq \frac{w^{H M}}{2} .
$$

Part (b) is implied by parts (a) and (c). To prove part (d), (22) implies that

$$
\frac{\mathrm{d}\left(t_{o}^{2}-t_{o}^{2}\right)}{\mathrm{d} \mu}=\frac{w}{4 n(1-\mu)^{2}(p-c)}>0 .
$$




\section{References}

Clemenz, G. 1990. "Non-Sequential Consumer Search and the Consequences of a Deregulation of Trading Hours." European Economic Review, 34: 1323-1337.

Clemenz, G. 1994. "Competition Via Shopping Hours: A Case for Regulation?" Journal of Institutional and Theoretical Economics, 150: 625-641.

Inderst, R., and A. Irmen. Forthcoming. "Shopping Hours and Price Competition." European Economic Review.

Kay, J., and C. Morris. 1987. "The Economic Efficiency of Sunday Trading Restrictions." Journal of Industrial Economics, 36: 113-129.

Neven, D. 1986. "On Hotelling's Competition with Non-Uniform Customer Distributions," Economics Letters, 21: 121-126.

Salop, S. 1979. "Monopolistic Competition with Outside Goods." Bell Journal of Economics, 10: $141-156$.

Shy, O., and R. Stenbacka. 2004. "Price Competition, Business Hours, and Shopping Time Flexibility," available for downloading from www. ozshy.com .

Stenbacka, R., and M. Tombak. 1995. "Time-Based Competition and the Privatization of Services," Journal of Industrial Economics, 43: 435-454.

Swan, p. 1970a. "Durability of Consumer Goods." American Economic Review, 60: 884-894.

Swan, p. 1970b. "Market Structure and Technological Progress: The Influence of Monopoly on Product Innovation." Quarterly Journal of Economics, 84: 627-638.

Tangay, G., L. Vallee and P. Lanoie. 1995. "Shopping Hours and Price Levels in the Retailing Industry: A Theoretical and Empirical Analysis." Economic Inquiry, 33: 516-524.

Thum, M., and A. Weichenrieder. 1997. "Dinkies and housewifes: The regulation of shopping hours." Kyklos, 50: 539-560.

Waterson, M. 1990. "Product Differentiation and Profitability: An Asymmetric Model," Journal of Industrial Economics, 39: 113-130. 
The Yrjö Jahnsson Working Paper Series in Industrial Economics is funded by The Yrjö Jahnsson Foundation.

\author{
Editor of the Working Paper Series: Professor Rune Stenbacka
}

2001

1. Anthony Dukes \& Esther Gal-Or: Negotiations and Exclusivity Contracts for Advertising

2. Oz Shy \& Rune Stenbacka: Partial Subcontracting, Monitoring Cost, and Market Structure

3. Jukka Liikanen, Paul Stoneman \& Otto Toivanen: Intergenerational Effects in the Diffusion of New Technology: The Case of Mobile

2002

1. Thomas Gehrig \& Rune Stenbacka: Introductory Offers in a Model of Strategic Competition

2. Amit Gayer \& Oz Shy: Copyright Protection and Hardware Taxation

3. Christian Schultz: Transparency and Tacit Collusion in a Differentiated Market

4. Luis H. R. Alvarez \& Rune Stenbacka: Strategic Adoption of Intermediate

Technologies: A Real Options Approach

5. Thomas Gehrig \& Rune Stenbacka: Information Sharing in Banking: An Anti-Competitive Device?

6. Oz Shy \& Rune Stenbacka: Regulated Business Hours, Competition, and Labor Unions

7. Jean Jacques-Laffont, Scott Marcus, Patrick Rey \& Jean Tirole: Internet Interconnection and The Off-Net-Cost Pricing Principle

8. Marc Ivaldi \& Frank Verboven: Quantifying the Effects from Horizontal Mergers in European Competition Policy

9. Ari Hyytinen \& Otto Toivanen: Do Financial Constraints Hold Back Innovation and Growth? Evidence on the Role of Public Policy

10. Staffan Ringbom \& Oz Shy: Advance Booking, Reservations and Refunds

2003

1. Luis H. R. Alvarez \& Rune Stenbacka: Irreversbility, Uncertainty and Investment in the Presence of Technological Progress

2. $\quad$ Thomas Gehrig \& Rune Stenbacka: Screening Cycles

3. Thomas Gehrig \& Rune Stenbacka: Differentiation-Induced Switching Costs and Poaching

4. Luis H.R. Alvarez \& Rune Stenbacka: Outsourcing or In-house Production? A Real Options Perspective on Optimal Organizational Mode

5. Staffan Ringbom \& Oz Shy: Reservations, Refunds, and Price Competition

6. Joanne Sault, Otto Toivanen \& Michael Waterson: Learning and Location

7. Tuomas Takalo \& Otto Toivanen: Entrepreneurship and Financial Markets with Adverse Selection

1. Oz Shy \& Rune Stenbacka : Liquidity Provision and Optimal Bank Regulation

2. Heikki Kauppi, Erkki Koskela \& Rune Stenbacka:Equilibrium Unemployment and Investment Under Product and Labour Market Imperfections

3. Mikael Juselius: The Relationship Between Wages and the Capital-Labor Ratio:

Finnish Evidence on the Production Function

4. Erkki Koskela \& Rune Stenbacka Agency Cost of Debt and Credit Market Imperfections: a Bargaining Approach

5. Luis H. R. Alvarez \& Rune Stenbacka: Takeovers and Implementation Uncertainty: A Real Options Approach

6. Oz Shy \& Rune Stenbacka: Service Hours With Asymmetric Distributions of Ideal Service Time 